\title{
Positive Emotions Are Not Simply the Absence of the Negative Ones: Development and Validation of the Emotional Recovery Questionnaire (EmRecQ)
}

\author{
Carolina Lundqvist and Göran Kenttä \\ Swedish School of Sport and Health Sciences
}

\begin{abstract}
The purpose of this study was to psychometrically evaluate the Emotional Recovery Questionnaire (EmRecQ) and to describe athletes' individual response patterns in five repeated assessments using the EmRecQ. Three samples were used. Samples 1 and 2 consisted of 192 and 379 (Mean age 16.4 years, $S D=0.7$ and Mean age: 17.0 years, $S D=1.1$ ) elite athletes from different sports. The third sample consisted of 20 (Mean age: $21.3, S D=19.0$ ) female elite basketball players. The EmRecQ is a 22-item questionnaire that assesses Happiness, Security, Harmony, Love, and Vitality. Results showed acceptable weighted omega reliability and construct reliability. Confirmatory factor analyses supported the a priori specified five-factor correlated model. Case profiles of repeated assessments revealed individual response patterns of the separate EmRecQ subscales that corresponded well with rated training load and total quality of recovery. The findings provide support for the EmRecQ's psychometric properties and applied usefulness.
\end{abstract}

It is important to increase our understanding of the recovery processes to enhance our ability to monitor health, well-being and performance in the population of athletes. Recovery has been defined as the psychosociophysiological process of eliminating fatigue and regaining vitality (Kenttä \& Dieffenbach, 2008). Conscious decisions and actions can alter the rate and configuration of the recovery process (Kellmann \& Kallus, 2001; Kenttä \& Hassmén, 1998). Athletes strive to choose the most valuable and efficient recovery activity from a wide range of alternatives; however, the activities per se only offer an incomplete understanding of recovery. Paying attention to emotional responses that arise from the nature of engagement in the activity will reveal important information regarding the quality of recovery (Sonnentag \& Fritz, 2007).

Emotions in sport have received increased attention in research and practice, with an emphasis on performance and well-being (e.g., Adie, Duda, \& Ntoumanis,

Lundqvist and Kenttä are with the Swedish School of Sport and Health Sciences, Stockholm, Sweden. 
2008; Hanin, 2000; Lavallee, Thatcher, \& Jones, 2005; Strean \& Strozzi-Heckler, 2009). Negative emotions have been studied a great deal in general psychology as well as in sport psychology. Positive emotions have received much less attention, which can be partly explained by the dominance of measures developed to assess negative emotional states (Jones, Lane, Bray, Uphill, \& Catlin, 2005). In the overtraining and recovery literature, mood states have generally been studied in preference to emotions. There is little consensus regarding the natures and definitions of emotion and mood. The majority of researchers do agree, however, that emotions are relatively short-lived reactions and are tied to real or appraised events or objects. Moreover, they are characterized by distinct cognitive and physiological changes such as altered subjective experiences and distinct facial expressions (e.g., Ekman, 1994; Fredrickson, 2005; Gross, 1999; Lazarus, 2000). Moods are generally referred to as longer-lasting and as more diffuse states not directed toward any specific goal (Fredrickson, 2005). The Profile of Mood States (POMS; McNair, Lorr, \& Droppelman, 1992) has been used extensively to assess mood disturbance as a response to negative overtraining (Urhausen \& Kindermann, 2002). Mood scores respond to changes in training load with a dose-response relationship. Consequently, decreased training load results in a decrease in negative mood scores in combination with an increase in vigor (i.e., displaying an "iceberg profile"; Raglin, 2001). More recently, mood scores have been used to monitor responses to both training and recovery (Kenttä, Hassmén, \& Raglin, 2006). Observing a decrease in negative mood states is important, but it may still be insufficient in providing a complete understanding of emotional recovery (Lazarus, 2000; Lucas, Diener, \& Larsen, 2003). Even though the POMS includes one positive mood state (vigor), it has limitations in assessing a full range of positive emotions that represent different functions in the recovery process. It would also be a limitation to conclude that recovery is completely satisfactory and functional based merely on the absence of overtraining markers. Considering a repertoire of different positive emotional processes may advance the understanding of how athletes regain energy and optimal functioning.

Various emotional states play an important role in terms of their capacity to either interfere with or enhance the process of recovery (Lucas et al., 2003; Sonnentag \& Fritz, 2007). Negatively toned emotions are generally characterized by a narrowing of an athlete's thought-action repertoire, high arousal and, if prolonged, energy depletion (Ekman, 1994; Fredrickson, 2005). Thus, negatively toned emotions are likely to interfere with a quick onset of an athlete's emotional recovery. For example, anger experienced as a result of athletes' casual attributions following poor performances was prolonged up to at least two days postcompetition (Allen, Jones, \& Sheffield, 2009). In contrast, positively toned emotional states have been associated with characteristics such as creativity, flexibility, relaxation, self-insight and reflexive thinking regarding life circumstances (Burns et al., 2008; Fredrickson, 1998, 2001; Fredrickson \& Branigan, 2005). These characteristics represent adaptive functions that broaden the athlete's cognitive thought-action repertoire and build durable personal and social resources that are important for the recovery process from a long-term perspective (Fredrickson, 2005). Hence, the primary functions of negative and positive emotional states appear to be fundamentally different. From a short-term perspective, Fredrickson and colleagues contend that positive emotions may actually have the potential to undo the detrimental effects of negative 
emotions. Empirical support for this "undoing hypothesis" has started to emerge in general psychology during the past decade (cf. Fredrickson, 2005; Fredrickson, Mancuso, Branigan, \& Tugade, 2000; Tugade \& Fredrickson, 2004).

Emotional regulations that cultivate athletes' positive emotions are likely to simultaneously promote functional recovery and prevent under-recovery. However, to assess and monitor recovery in athletes there are only two instruments available in the scientific literature, neither of which was developed to assess the emotional aspect of recovery. The Recovery-Stress Questionnaire for Athletes (RESTQ; Kellmann \& Kallus, 2001) simultaneously assesses stressful and restful events during a period of three days/nights. More specifically, the general recovery scales assess success, social recovery, physical recovery, general well-being, and sleep quality. The sport-specific recovery scales assess being in shape, personal accomplishment, self-efficacy, and self-regulation. The Total Quality Recovery Scale (TQR; Kenttä \& Hassmén, 1998) assesses recovery actions and global perceptions of recovery on a daily basis. It is questionable whether the existing recovery instruments, in their present form, are suitable for monitoring emotional time-to-time changes of brief duration. Perhaps most importantly, there has been a total lack of instruments that focus on positive emotions related to recovery. The purpose of this study was therefore to develop and psychometrically evaluate a new measure of emotional recovery, the EmRecQ. Another aim was to describe athletes' response patterns of repeated assessments of the EmRecQ in relation to training load and total quality of recovery.

\section{Method}

\section{Participants}

The participants in the initial sample consisted of 192 athletes (87 men and 105 women) with a mean age of 16.4 years $(S D=0.7)$ who were actively involved in competitive sports. All participants were students at specifically designated sport high schools in Sweden whose competitive level ranged from national elite to international junior elite. The sports represented in the sample were soccer $(n=$ $66)$, handball $(n=40)$, basketball $(n=34)$, ice hockey $(n=20)$, equestrian sport $(n$ $=11)$, American football $(n=7)$, floor-ball $(n=5)$, swimming $(n=3)$, track and field sports $(n=1)$, gymnastics $(n=1)$, and cycling $(n=1)$. Three athletes did not specify their sport.

To replicate the findings and to analyze the invariance of the factor structure across gender, data were collected from a second sample of sport high school students. The participants were 379 athletes (168 men and 211 women) who were an average of 17.0 years of age $(S D=1.1)$ and competed at national and international junior elite levels. Sports represented in the sample were skiing (biathlon $n=25$, downhill $n=33$ and cross-country $n=49)$, basketball $(n=47)$, soccer $(n$ $=91)$, track and field sports $(n=12)$ and ice-hockey $(n=63)$, and 59 athletes did not specify their sport.

Finally, a third sample consisting of 20 female elite basketball players was used to evaluate individual emotional recovery profiles in relation to total quality of recovery and training load over a period of ten weeks. All participants were actively playing in the highest-level basketball league in Sweden and were an 
average of 21.3 years of age $(S D=0.9)$. Due to illness, a few participants failed to complete all five assessments (assessment no. $1=3$ missing, no. $2=4$ missing, no. $3=1$ missing, no. $4=0$ missing, no. $5=1$ missing). Of these, one participant was missing at two assessments and the remaining participants who failed to complete all EmRecQ assessments were absent at one assessment.

\section{Instrument}

Development of the EmRecQ: The EmRecQ was developed using both an empirically and a theoretically driven approach. Just over 300 competitive athletes and coaches with a prior athletic career, who were participating in sport studies classes or seminars at university level, were asked to provide three elucidating emotional expressions associated with functional recovery. The data were reduced to a set of suitable items by excluding expressions not referring to emotions per se according to scientific definitions (e.g., Ekman, 1994; Fredrickson, 2005; Lazarus, 2000) and expressions assigned by merely one or a few participants. Conceptually similar emotional expressions were grouped together as they were judged to refer to the same emotional states. During this qualitative phase of scale development, two individual coders (the authors) were used to control for possible biases, and the categorization and labeling of emotional dimensions were discussed until consensus was reached. Finally, a qualitative item analysis was performed with athletes on one Olympic team who confirmed the suitability and clarity of the items and the scale's applicability for monitoring recovery in an applied setting.

The Final Version of the EmRecQ: The EmRecQ, displayed in its full version in Appendix 1, consists of 22 items that assess five emotional states. Answers are rated on a five-point scale ranging from "Not at all" to "Extremely". The five subscales are labeled "Happiness" (4 items), "Security" (4 items), "Harmony" (5 items), "Love" (4 items), and "Vitality" (5 items).

Happiness is defined as elicited by the effective usage of physical and mental resources and the subjective experience of an individual advancing toward significant goal(s) in his/her life (Lazarus, 2000). It is linked with participation in activities perceived as interesting and meaningful in relation to the athlete's skill level (e.g., Nakamura \& Csikszentmihalyi, 2005). Happiness is also related to health status, motivation, creativity, involvement and high levels of performance (Fredrickson, 2005; Keyes \& Magyar-Moe, 2002).

Security is defined as a subjectively experienced stability, surety and control with respect to present and future situations and significant others (Soares, Lemos, $\&$ Almeida, 2005). Security in the self and toward others is a central part of attachment and the building of relationships (Amitay \& Mongrain, 2007; Soares et al., 2005), and of the perception of the availability of social support resources to cope with or buffer against stressful circumstances (Bianco \& Eklund, 2001).

Harmony refers to a nonstressful experience, characterized by a feeling of inner calmness, balance and contentment, in which the need for and value of recovery are internalized and freely accepted by the athlete. Harmony helps the athlete unwind from experiences of stress and prompts him/her to restful self-insight and reflection on life circumstances (Fredrickson, 2005; Sonnentag \& Fritz, 2007).

Love is defined as unconditional "friendship love". It represents the positive liking between people such as teammates, coaches, family members or friends who 
share significant parts of their lives (Henderick \& Henderick, 2005). Moreover, it is experienced without any performance or earning demands attached to it (Gould, Lauer, Rolo, Jannes, \& Pennisi, 2006). Love is fundamental to the perception of relatedness (Deci \& Ryan, 2000) and is strongly associated with internalized motives, health and well-being.

Vitality is defined as the subjective experience of having access to physical and mental energy (Ryan \& Frederick, 1997) and as self-regulated energy that could be used for purposive actions (Ryan \& Deci, 2008). Moreover, it is related to physical factors such as immunological functioning, sleep, diet and training loads, and to psychological factors such as experienced demands, gratification, coping and intrinsic motivation (Nix, Ryan, Manly, \& Deci, 1999; Ryan \& Deci, 2008; Ryan \& Frederick, 1997).

For the data collection from the third sample, two additional instruments were used: The Total Quality Recovery Scale (TQR; Kenttä \& Hassmén, 1998; 2002) and the single item CR10 scale (Borg, 1998). The TQR scale has a structure similar to the perceived exertion scale, but has the purpose of assessing recovery based on a single global self-rating of all available psychophysiological cues.

\section{Procedures}

When Samples 1 and 2 were collected, the purpose of the study was explained to the staff at each sport high school. After their consent, surveys were distributed in classroom settings in close collaboration with the responsible school staff. The third sample was assessed in collaboration with the head coach and physiotherapist at weekly team meetings for four consecutive weeks before the start of the competitive season, and once six weeks into the season, a total of five assessments for a period of ten weeks. Before data collection, all participants were provided with written and oral information regarding the confidentiality of results, the voluntariness of taking part in the study, and the possibility to discontinue participation at any time without consequences. All procedures were in accordance with national and APA ethical guidelines, and all participants gave written informed consent before completing the inventory.

\section{Statistical Analyses}

Confirmatory factor analyses (CFA) using maximum likelihood estimation were performed using EQS 6.1 (Bentler, 1995). All measurement models tested were over-identified according to the t-rule and the three-indicator rule (Bollen, 1989). Because the normalized estimate of Mardia's coefficient was significant (Sample 1: 17.58 and Sample 2: 23.40), indicating multivariate nonnormality in data, SatorraBentler's scaled statistics were used in subsequent analyses to produce robust chi-square ( $\mathrm{S}-\mathrm{B} \chi^{2}$ ) statistics and robust standard errors. To evaluate goodness of fit, several goodness-of-fit indices were used, including the scaled chi-square (S-B $\chi^{2}$ ), the comparative fit index (CFI), the nonnormed fit index (NNFI), the root mean square error of approximation (RMSEA) including the $90 \%$ confidence interval $(90 \% \mathrm{CI})$, and the standardized root mean square residual (SRMR). For the CFI and the NNFI, values above .90 are traditionally considered reasonable model fit, whereas newer recommendations suggest values close to .95 as more 
reliable (CFI; Bentler, 1995; Hu \& Bentler, 1999). For the RMSEA and the SRMR, values of .08 and below are suggested to indicate reasonable fit (Brown \& Cudek, 1993; Hu \& Bentler, 1998, 1999). The factorial invariance of the EmRecQ across gender in Sample 2 was evaluated using a multistep approach as recommended by Byrne (2006), in which chi-square difference and CFI difference were evaluated between the constrained models tested. Finally, the results from the third sample were displayed descriptively to visualize individual profiles of athletes' recovery and training load patterns.

\section{Results}

\section{Initial Analyses in Sample 1}

Interfactor correlations, mean values and standard deviations for the subscales of Sample 1 are displayed in Table 1. Prior research indicates that diverse positive emotions are strongly related (Lucas et al., 2003). Consequently, even though the EmRecQ was developed as a five-factor model it was theoretically supported to also evaluate the presence of a second-order factor. Thus, two measurement models were evaluated: Model 1 was the theoretically a priori specified five-factor correlated model, and Model 2 was the theoretically a priori second-order model with all first-order factors specified to load onto one single second-order factor. All parameter estimates in the analyses were significant $(t>1.96)$. Although the chi-square statistics were significant $\left(\mathrm{S}-\mathrm{B} \chi^{2}(199)=345.64, p<.001\right)$, Model 1 displayed a reasonable model fit $(\mathrm{CFI}=.93, \mathrm{NNFI}=.92, \mathrm{SRMR}=.06, \mathrm{RMSEA}$ $=.06(90 \% \mathrm{CI}=.05-.07))$. A similar model fit was revealed when Model 2 was evaluated $\left(\mathrm{S}-\mathrm{B} \chi^{2}(204)=352.46, p<.001, \mathrm{CFI}=.93, \mathrm{NNFI}=.92, \mathrm{SRMR}=\right.$ $.06, \mathrm{RMSEA}=.06(90 \% \mathrm{CI}=.05-.07))$. A subsequent chi-square difference test $\left(\mathrm{S}-\mathrm{B} \chi_{\text {diff }}^{2}(5)=6.82, p=\right.$ n.s. $)$ and CFI difference (.00) confirmed that a second-order factor did not improve model fit. Based on the results of the model comparison and theoretical support for the five correlated latent factors, Model 1 was regarded as an adequate target model. Model 2 was, however, statistically equivalent to Model 1.

As shown in Table 2, the standardized solution for factor loadings (range: .62-.81), error variances (range: .35-.62) and squared multiple correlations (SMC, range: .38-.66) for Model 1 indicated that the items were adequate indicators of the latent factors. Reliability estimations based on unequal weighting have been suggested as most appropriate following a CFA (Bacon, Sauer, \& Young, 1995); thus composite reliability was computed in terms of weighted omega coefficients for the five factors. Results showed acceptable weighted omega scores for all subscales (Happiness $\Omega_{w}=.81$, Security $\Omega_{w}=.83$, Harmony $\Omega_{w}=.85$, Love $\Omega_{w}$ $=.83$, and Vitality $\Omega_{w}=.84$ ). Moreover, construct reliability based on the formula by Fornell and Larcker (1981) was computed. The construct reliability estimates describe the variance captured by measurement errors as opposed to the variance attributable to the latent factors. A coefficient less than .50 indicates that the error variance is greater than the variance of the constructs. The results revealed construct coefficients that ranged from .49 to .54 (Happiness $=.51$, Security $=.54$, Harmony $=.53$, Love $=.53$, and Vitality $=.49$ ). 


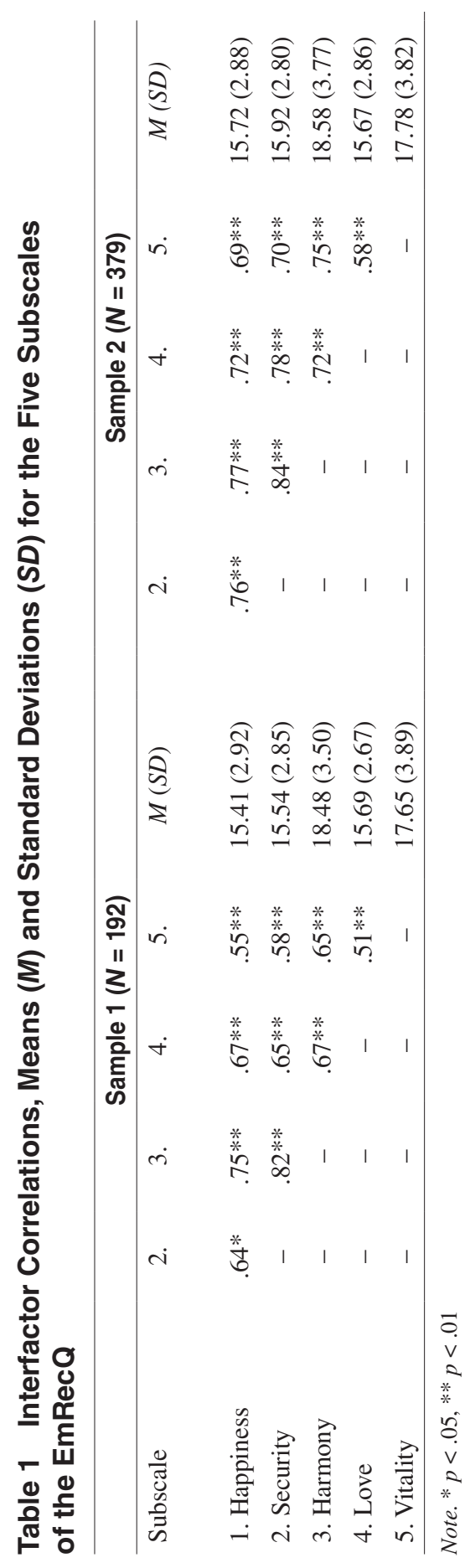




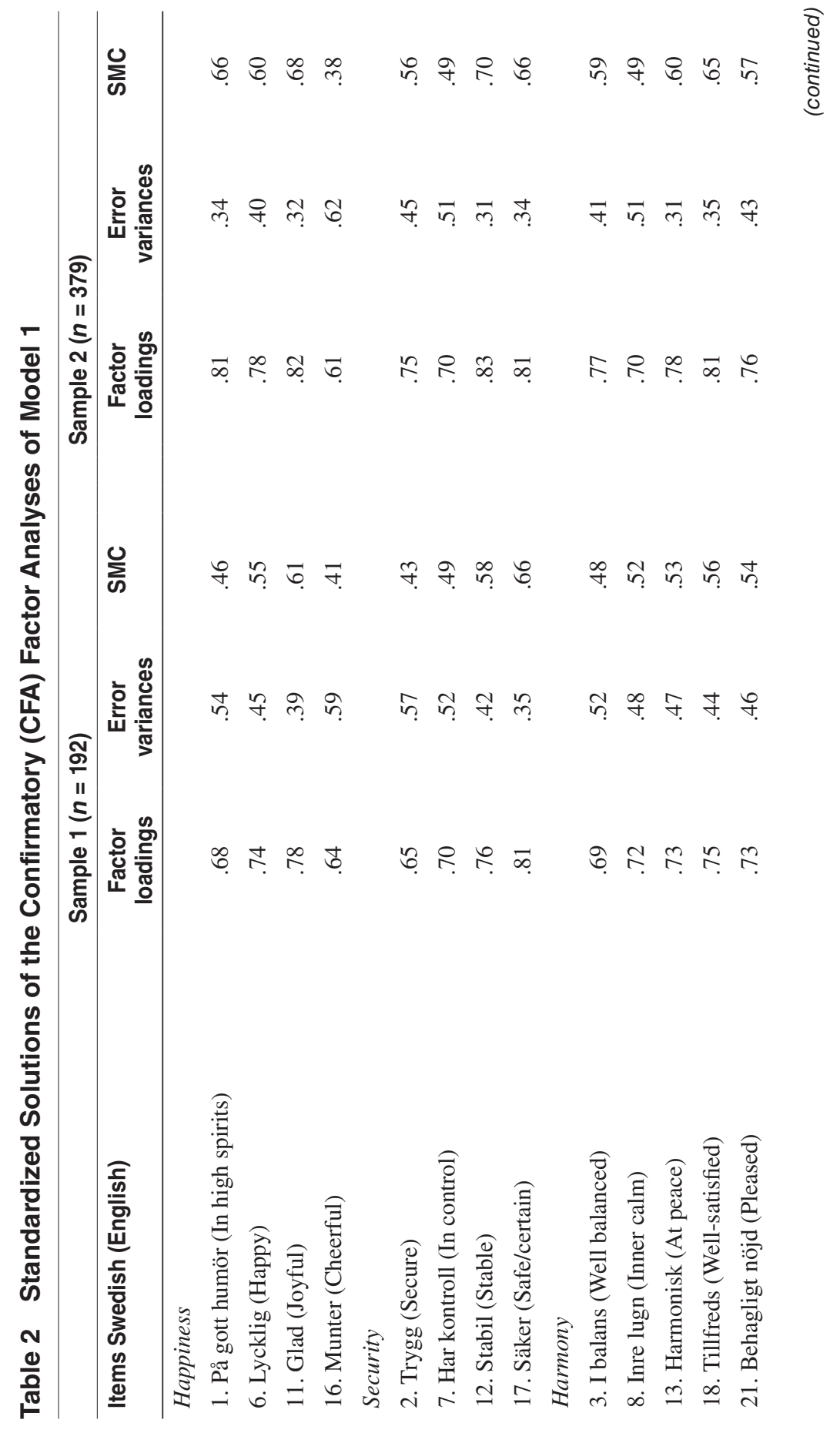




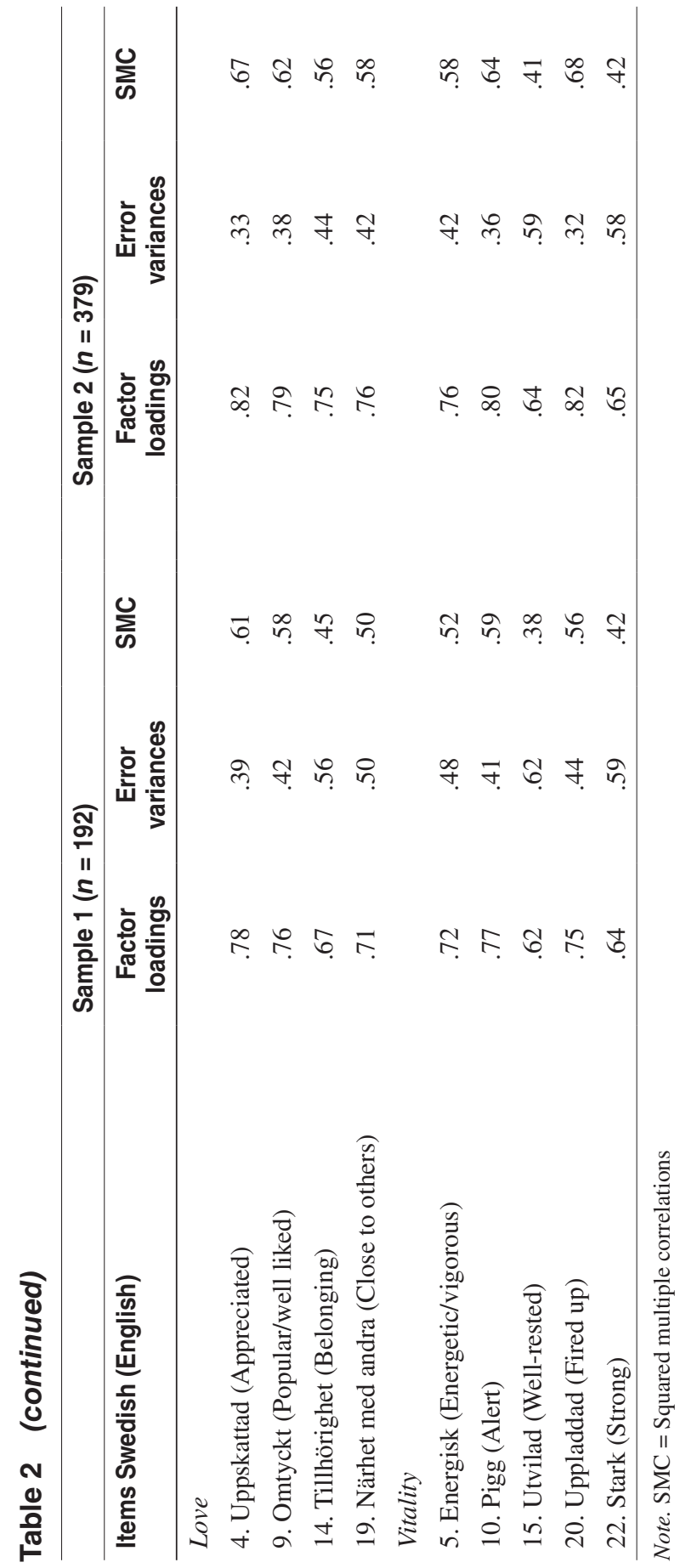




\section{Replicating Analyses and Invariance Tests Across Gender in Sample 2}

To replicate the analyses of Models 1 and 2 and also to perform invariance tests across gender, a large independently collected sample was used. Interfactor correlations, mean values and standard deviations for Sample 2 are shown in Table 1. The CFA conducted on the entire sample showed a reasonable model fit for Model 1 $\left(\mathrm{S}-\mathrm{B} \chi^{2}=517.39(199), p<.001, \mathrm{CFI}=.91, \mathrm{NNFI}=.90, \mathrm{SRMR}=.05, \mathrm{RMSEA}=\right.$ $.07(90 \% \mathrm{CI}=.06-.07))$. Model 2 showed a somewhat decreased fit in terms of an increased chi-square value and a decreased NNFI value (S-B $\chi^{2}=551.78(204), p$ $<.001, \mathrm{CFI}=.91, \mathrm{NNFI}=.89, \mathrm{SRMR}=.05, \mathrm{RMSEA}=.07(90 \% \mathrm{CI}=.06-.07)$ ). A chi-square difference test $\left(\mathrm{S}-\mathrm{B} \chi_{\text {diff }}^{2}(5)=34.39, p<.001\right)$ and CFI difference $\left(\mathrm{CFI}_{\text {diff }}=.01\right)$ also indicated a decreased fit of Model 2 when compared with Model 1. Thus, Model 1 was still regarded as the target model while it was acknowledged that the differences between Models 1 and 2 were rather small. The standardized solution for Model 1 is shown in Table 2. As displayed, factor loadings (range: $.61-.83$ ), error variances (.31-.62) and SMS (range: .38-.70) suggested that the items were adequate indicators of the latent factors. Weighted omega reliability scores for the factors were all at acceptable levels (Happiness $\Omega_{w}=.86$, Security $\Omega_{w}$ $=.86$, Harmony $\Omega_{w}=.88$, Love $\Omega_{w}=.86$, and Vitality $\left.\Omega_{w}=.87\right)$, as were construct reliability coefficients (Happiness $=.58$, Security $=.60$, Harmony $=.59$, Love $=$ .61 , and Vitality $=.55)$.

Gender Invariance Analyses: The measurement factorial invariance across gender was evaluated using a multistep procedure as recommended by Byrne (2006). Model 1 demonstrated an adequate model fit among the separate samples of men $\left(\mathrm{S}-\mathrm{B} \chi^{2}=354.46(199), p<.001, \mathrm{CFI}=.90, \mathrm{NNFI}=.88, \mathrm{SRMR}=.06\right.$, RMSEA $=.07(90 \% \mathrm{CI}=.06-.08))$ and women $\left(\mathrm{S}-\mathrm{B} \chi^{2}=390.26(199), p<\right.$ $.001, \mathrm{CFI}=.91, \mathrm{NNFI}=.90, \mathrm{SRMR}=.05, \mathrm{RMSEA}=.07(90 \% \mathrm{CI}=.06-.08)$, even though NNFI was slightly below the cut-off value for the sample of men. As displayed in Table 3, model fit was still adequate when invariance constraints were placed stepwise on factor loadings, covariances and error variances. The CFI showed no difference $\left(\mathrm{CFI}_{\text {diff }}=.00\right)$ between the baseline model and the subsequently more constrained models and the chi-square difference was nonsignificant, supporting the invariance of the factor structure across gender. When Model 2 was analyzed, the results displayed a somewhat decreased fit to data compared with Model 1 for both men (S-B $\chi^{2}=374.35$ (204), $p<.001$, CFI $=.89, \mathrm{NNFI}=.88, \mathrm{SRMR}=.07, \mathrm{RMSEA}=.07(90 \% \mathrm{CI}=.06-.08)$ and women $\left(\mathrm{S}-\mathrm{B} \chi^{2}=412.44(204), p<.001, \mathrm{CFI}=.90, \mathrm{NNFI}=.89, \mathrm{SRMR}=.05, \mathrm{RMSEA}\right.$ $=.07(90 \% \mathrm{CI}=.06-.08))$. A chi-square difference test supported that Models 1 and 2 differed significantly for both men and women $\left(\right.$ Men: $S-B \chi_{\text {diff }}^{2}(5)=19.89$, $p<.001$ and Women: $\left.\mathrm{S}-\mathrm{B} \chi_{\text {diff }}^{2}(5)=22.18, p<.001\right)$, whereas CFI difference only indicated a decreased fit for the sample of women (Men: $\mathrm{CFI}_{\text {diff }}=.00$ and Women: $\mathrm{CFI}_{\text {diff }}=.02$ ). In Table 3, the results from the invariance test of Model 2 across gender are displayed. The chi-square difference test and $\mathrm{CFI}\left(\mathrm{CFI}_{\text {diff }}=\right.$ $.00)$ showed that Model 2 was invariant across gender when factor loadings and error variances were constrained stepwise. 


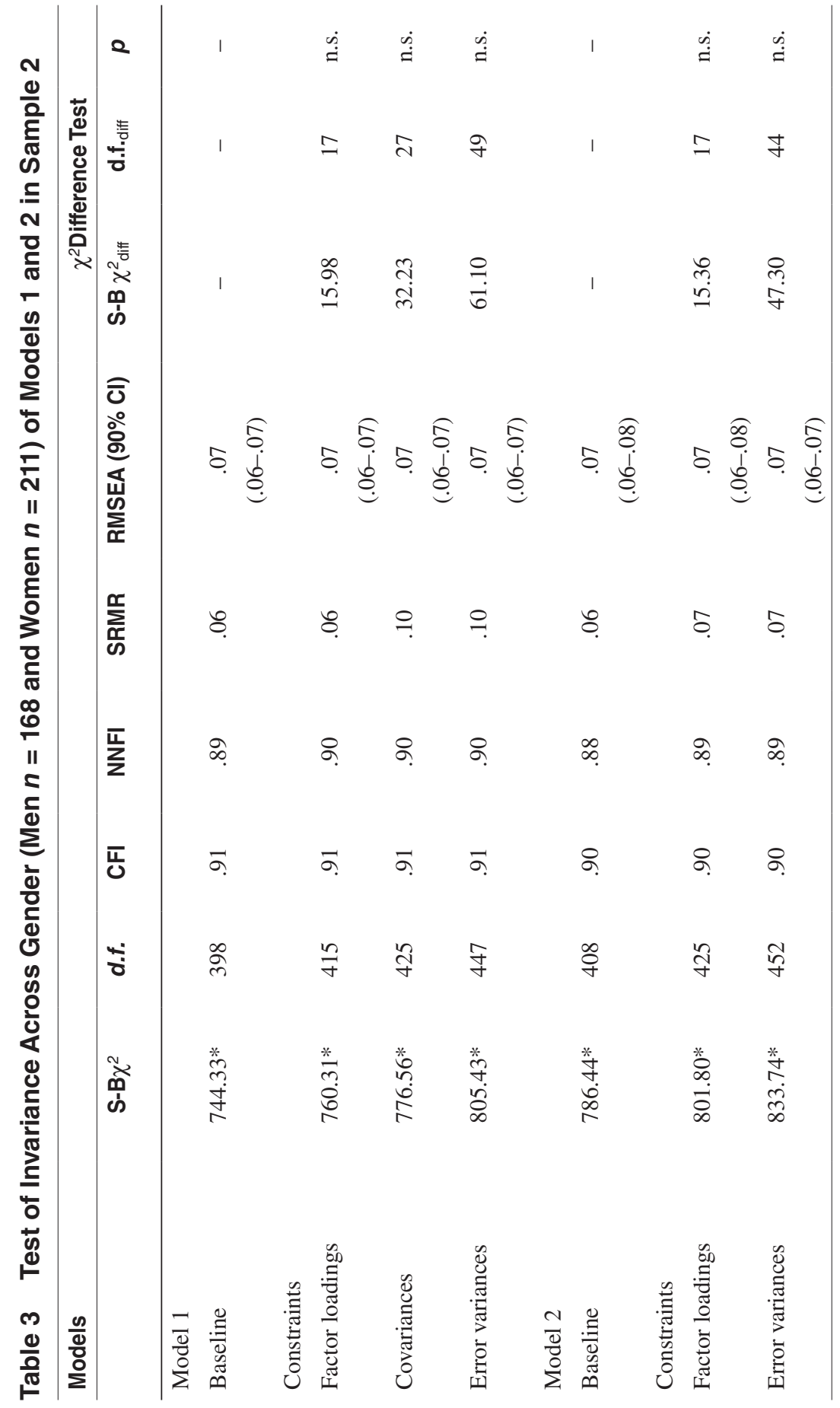




\section{Individual Profiles of Recovery and Training Load in Sample 3}

To evaluate individual patterns of recovery and training load, case profiles were summarized. Three of the 20 athletes were selected based on their diverse TQR scores: Athletes 1 and 2 displayed the lowest initial scores on the TQR (TQR = 2 and 1, respectively). The TQR score of Athlete 1 increased slightly over the following assessments, whereas that of Athlete 2 varied greatly. Athlete 2 was not present during the fifth assessment occasion due to illness, resulting in four assessments for this athlete. Athlete 3 displayed the highest initial TQR score $(=8)$, which increased and then decreased slightly over time. In Figures 1-3 the EmRecQ subscales, EmRecQ total scores, TQR scores and CR10 assessment of the week's total training load are displayed as individual profiles of these three athletes. In Figure 4, the profile of the total sample $(n=20)$ is displayed. As can be seen in Figures 1-4, the pattern of the TQR scores and the global scores of the EmRecQ follow very similar patterns in both the individual cases and the group profile. Inspecting the diverse subscales of the EmRecQ in the individual cases, great individual variations are displayed in terms of the different patterns of the five emotional ratings. In Table 4, Pearson product moment correlation coefficients between the EmRecQ, TQR and CR10 scores are displayed. Albeit the sample size was small and thereby decreases the power in the analyses, the results indicate that the EmRecQ subscales are positively related to recovery scores. A less consistent pattern is shown regarding the relationship between EmRecQ subscales and training load scores.

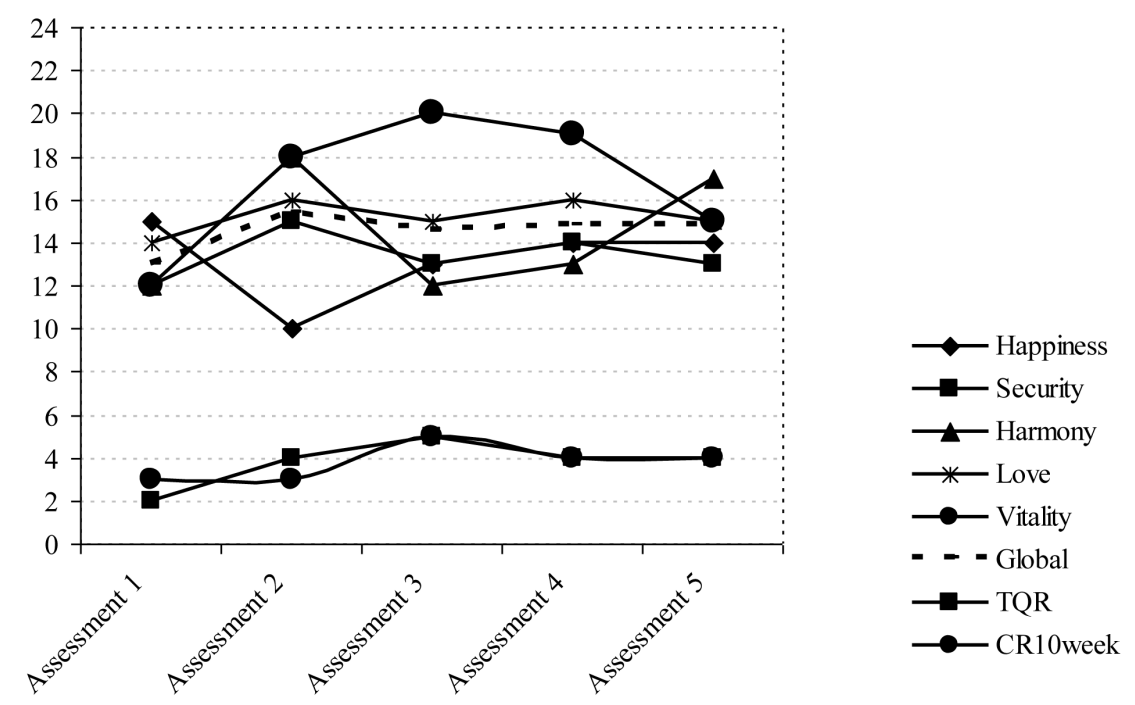

Figure 1 - Individual case profile of lowest initial and increasing TQR scores of the sample together with individual EmRecQ and CR-10 weekly training load scores. 


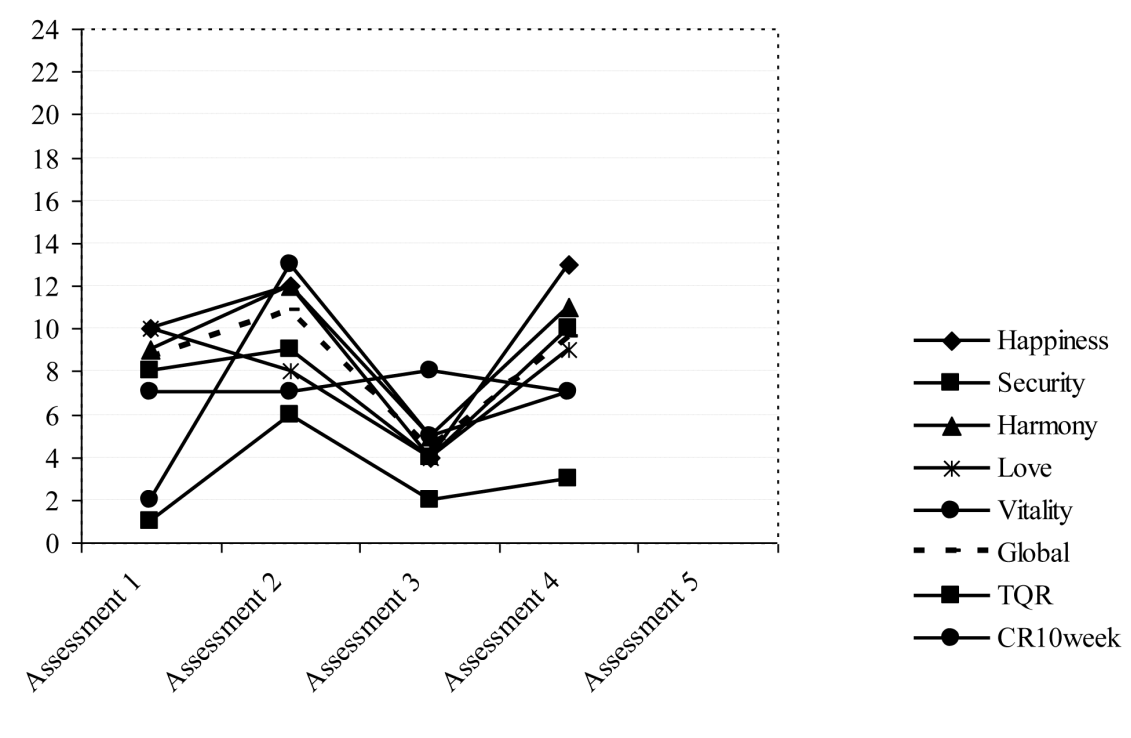

Figure 2 - Individual case profile of the lowest initial and varying TQR scores of the sSample together with individual EmRecQ and CR-10 weekly training load scores.
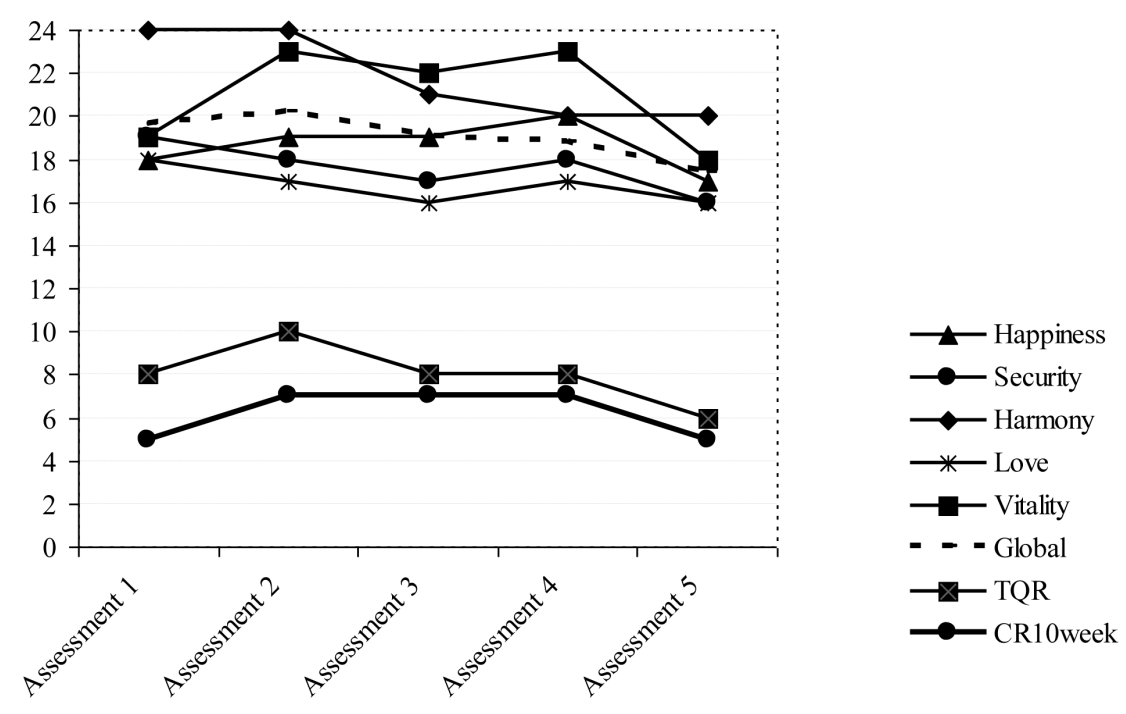

Figure 3 - Individual case profile of the highest initial TQR scores of the sample together with individual EmRecQ and CR-10 weekly training load scores. 


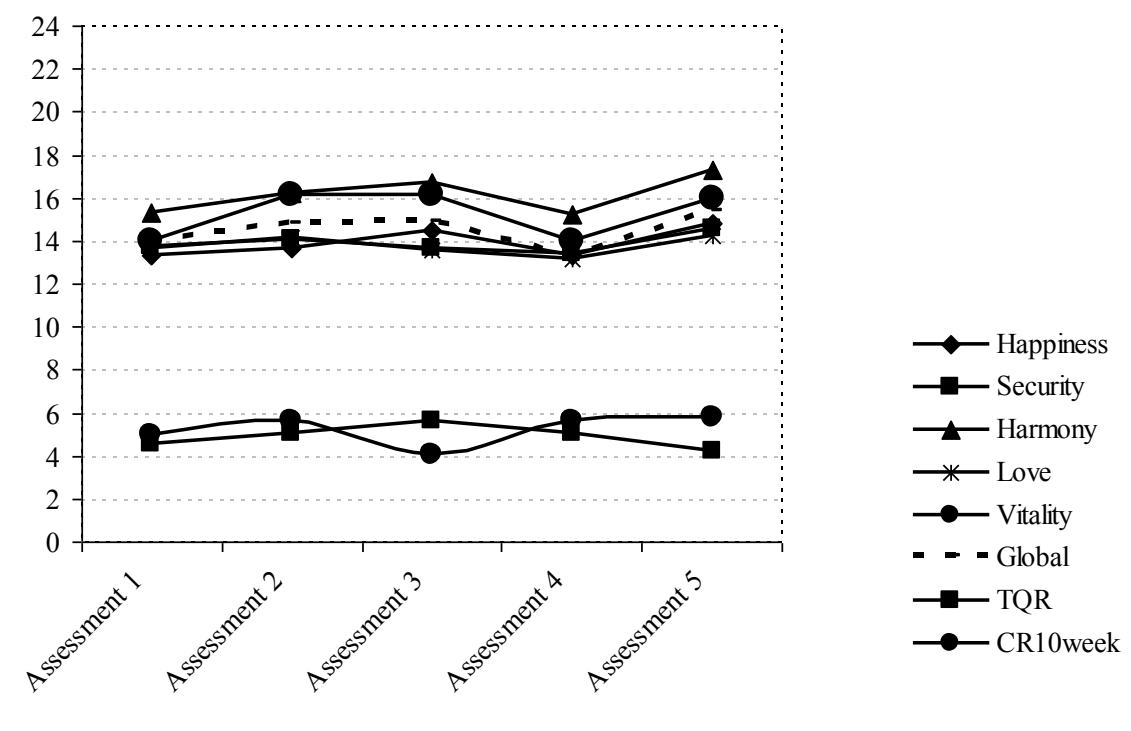

Figure 4 - Profile of the total sample's $(n=20)$ EmRecQ, TQR and CR-10 training load ratings.

\section{Discussion}

A range of emotional questionnaires has been developed and used in the sport psychology research literature during recent decades, but there is still a need for advances in scales for assessing positively toned emotional states. Progress in the theoretical understanding of emotional states related to fatigue or energy, and thus optimal recovery, is largely dependent on an ongoing improvement in the sophistication of the measurements used (O'Connor, 2004). The EmRecQ presented in this article was developed to overcome some of this questionnaire sparseness, with a primary focus on advancing the assessment of positive emotional states experienced in relation to athletes' recovery. The results of this psychometric evaluation indicate that the EmRecQ is a promising questionnaire. The reliability of the scale was supported in terms of weighted omega reliability and construct reliability coefficient. The CFA supported the factorial validity of the scale as the a priori hypothesized five-factor model revealed an acceptable model fit. The five-factor model was also invariant across gender. Thus, throughout all analyses of the global model fit, the results indicated support for the a priori specified five-factor correlated model. The individual case profiles revealed that athletes displayed various response patterns of the five subscales of the EmRecQ when related to their overall recovery and training loads. These results support the applied value of the five-factor model of the EmRecQ to monitor the individual emotional response to recovery and training load over time. Moreover, these results support that the underlying emotional processes of recovery are multifaceted and that diverse positive emotional responses might be present among athletes. Monitoring of athletes' emotional processes over time provides increased opportunities to tailor adequate individual recovery strategies based on their unique emotional profiles. 


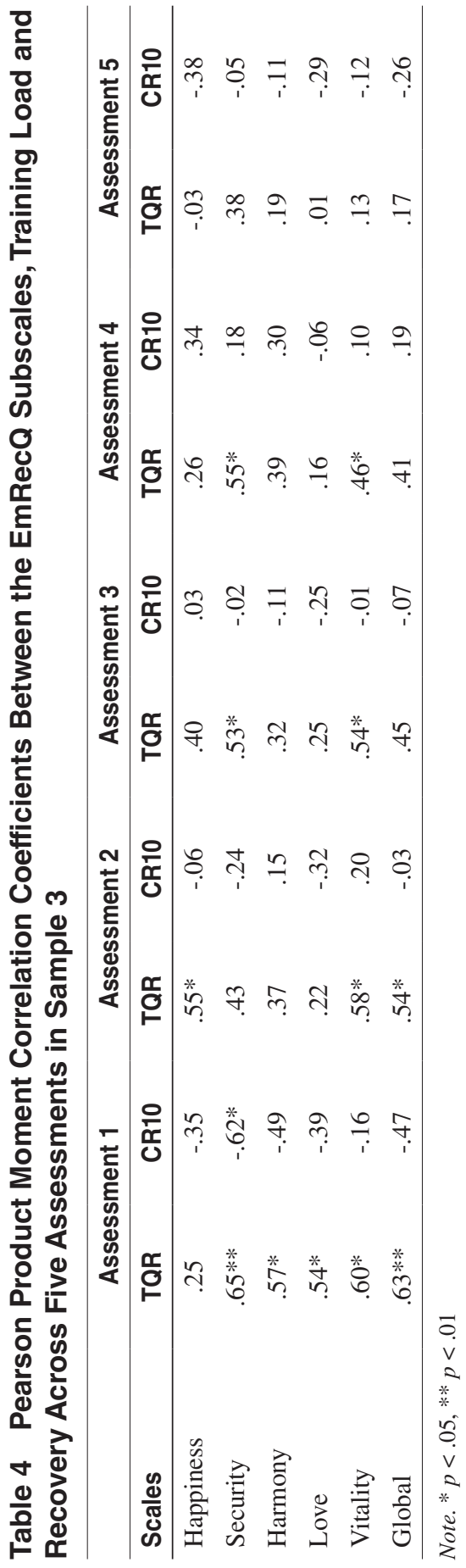


Although the psychometric properties and the applied usefulness of the fivefactor model of the EmRecQ were supported by the results, the second-order factor model displayed only a slightly decreased model fit compared with the five-factor model. An unexpected finding was also that the global score of the EmRecQ displayed a pattern that was very similar to the TQR score in both the individual case profiles and the group profile of Sample 3. Based on these results, it seems plausible that the global score of the EmRecQ could be an easily accessible indicator of athletes' overall state of psychological recovery. Future research is warranted to further investigate the usefulness of the global score of the EmRecQ in comparison with well-established psychological recovery indicators.

The results presented in this article were an initial step in the validation process of the questionnaire. Validation of any instrument is an ongoing process, however. Further empirical investigation and replication in independently drawn samples of various sports and age groups are therefore warranted. In addition, although it has been suggested that basic emotions are relatively universal in nature (e.g., Ekman, 1994), we do not argue that the emotional states presented in the EmRecQ are basic in such a strict sense. Instead, they represent theoretically reinforced emotional states, assessed with emotional adjective items identified by Swedish athletes and coaches as characteristic of their optimal recovery. Researchers wanting to use the EmRecQ in a cross-cultural perspective should therefore explore whether the emotional states included are applicable across different cultures. To enable cross-cultural research, a translated English version of the EmRecQ is presented in the appendix of this article. Some caution is called for, however, as preclusion of linguistic differences across the Swedish and translated English versions have not yet been confirmed in any published study. Nevertheless, the authors' unpublished data provide preliminary support for the psychometric soundness of the translated English version.

Even though the supported a priori hypothesized five-factor structure of the EmRecQ provides initial support for the scale's construct validity, conclusions regarding construct validity should be based on a number of empirical findings that together provide support for theoretical presumptions that are in line with the hypotheses $(\mathrm{Li}$ \& Harmer, 1996). Convergent and discriminant validity evidence, in terms of support for anticipated relationships between the EmRecQ subscales and theoretically similar or dissimilar constructs, is now also warranted. Data collection using the EmRecQ and additional affective, recovery and subjective well-being assessments, such as the POMS, the RESTQ and the Satisfaction with Life Scale (SWLS; Diener, Emmons, Larsen, \& Griffin, 1985), could be a practicable course for obtaining such evidence.

Within the framework of positive psychology, the importance of positive emotional states is now highly emphasized. Positive emotions are known to fill an important function as indicators of well-being and optimal functioning. There is also a growing body of evidence supporting that interventions aimed at fostering positive emotions may enhance well-being and health over time. As postulated by the broaden-and-build theory of positive emotions (Fredrickson, 1998, 2001, 2005), negative emotions are known to narrow the cognitive capacity of the individual. Positive emotions appear instead to broaden the cognitive capacity as well as increase optimal and flexible cognitive functioning. Moreover, according to the undoing hypothesis, it is suggested that positive emotions diminish negative emotions and the detrimental effects they may cause (cf. Fredrickson, 2005; Fredrickson et al., 2000; Tugade \& Fredrickson, 2004). The broadening mechanism of positive emotions may 
therefore prove important in facilitating a more rapid recovery among athletes who experience negative emotions. These theoretical suggestions are based primarily on empirical evidence displayed in laboratory studies within general psychology. Further research on athlete populations that examines ecological validity in a sports context is therefore highly warranted. If supported, emotion-regulation strategies should be designed specifically for the stimulation of positive emotions to help athletes undo the outlasting effects of negative emotions. Moreover, such findings would indeed emphasize the need in athletic recovery research literature to carefully assess the balance of both negative and positive emotional states related to athletes' overall well-being and health.

Monitoring the balance between training and recovery still remains one of the greatest unresolved challenges in the applied setting of elite sports concerning athletes, coaches, and scientists (Kenttä et al., 2006; Main \& Groove, 2009). This is the case particularly during heavy training, when the dominant approach has focused on assessing stress load (i.e., the training stimulus) and short-term fatigue responses. In contrast, the recovery process has received limited attention, most likely due to the lack of instruments developed to monitor recovery (Kenttä et al., 2006). We believe that the EmRecQ has the potential to shift the focus from primarily monitoring physiological stress. Consequently, it provides a means for competitive athletes to enhance the monitoring of adequate and functional recovery and thereby increase the possibility to prevent underperformance and maladaptive responses to training.

An additional plausible area of application for the EmRecQ, well worth further contemplation, is exercise adherence and individuals' engagement in healthy lifestyles. In research literature, the translation of individuals' intentions to exercise into actual behavior of physical activity has proven problematic, and behavior is still unsatisfactorily predicted by traditional models like the Theory of Planned Behavior (Mohiyeddini, Pauli, \& Bauer, 2009). A possible cause of this exercise intention-exercise behavior gap suggested in research literature is that emotions and related affective processes are not explicitly included in the models. Indeed, a recent study (Mohiyeddini et al., 2009) testing this hypothesis revealed that inclusion of emotions in the model increased the explained variance of exercise duration and frequency by 20 and $17 \%$, respectively. Moreover, positive emotions proved to influence the intention to exercise, suggesting that emotionally based interventions may be adaptive in increasing exercise participation. Mohiyeddini and colleagues (2009), however, assessed positive emotions using selected vigor adjectives from the POMS-C (Lane \& Terry, 1998), an approach probably used as a consequence of the lack of available assessments designed to assess positive emotions. Expanding this line of research using the EmRecQ, or an exercise-adapted version of it, could therefore provide new insight into important processes that generate exercise adherence and related health behaviors.

In conclusion, the EmRecQ shows promising psychometric results and can be used as a complementary measure with already established instruments such as the POMS. Moreover, the EmRecQ has a number of plausible areas of application within sport and exercise psychology, and offers a unique contribution to the research literature due to its focus on positive emotional states. Although the current study displayed support for the psychometric properties of the scale, further reliability and validity evidence collected in independently drawn samples is highly warranted. 


\section{Acknowledgments}

This study was made possible by research grants from the Swedish National Centre for Sports Research and the Swedish School of Sport and Health Sciences

\section{References}

Aide, J.W., Duda, J.L., \& Ntoumanis, N. (2008). Achievement goals, competition appraisals, and the psychological and emotional welfare of sport participants. Journal of Sport \& Exercise Psychology, 30, 302-322.

Allen, M.S., Jones, M.V., \& Sheffield, D. (2009). Casual attribution and emotion in the days following competition. Journal of Sports Sciences, 27, 461-468.

Amitay, O.A., \& Mongrain, M. (2007). From emotional intelligence to intelligent choice of partner. The Journal of Social Psychology, 147, 325-343.

Bacon, D.R., Sauer, P., \& Young, M. (1995). Composite Reliability in Structural Equations Modeling. Educational and Psychological Measurement, 55, 394-406.

Bentler, P.M. (1995). EQS Structural Equations Program Manual. Encino, CA: Multivariate Software, Inc.

Bianco, T., \& Eklund, R.C. (2001). Conceptual considerations for social support research in sport and exercise settings: The case of sport injury. Journal of Sport \& Exercise Psychology, 23, 85-107.

Bollen, K.A. (1989). Structural equations with latent variables. New York: Wiley.

Borg, G. (1998). Borg's perceived exertion and pain scales. Champaign, IL: Human Kinetics.

Browne, M.W., \& Cudek, R. (1993). Alternative ways of assessing model fit. In K.A. Bollen \& J.S. Long (Eds.), Testing Structural Equation Models (pp. 136-162). Newbury Park, CA: Sage.

Burns, A.B., Brown, J.S., Sachs-Ericsson, N., Plant, E.A., Curtis, J.T., Fredrickson, B.L., et al. (2008). Upward spirals of positive emotions and coping: Replication, extension, and initial exploration of neurochemical substrates. Personality and Individual Differences, 44, 360-370.

Byrne, B.M. (2006). Structural equation modelling with EQS. Basic concepts, applications, and programming (2nd ed.). London: Lawrence Erlbaum Associates, Publishers.

Deci, E.L., \& Ryan, R.M. (2000). The "what" and "why" of goal pursuits: Human needs and the self-determination of behavior. Psychological Inquiry, 11, 227-268.

Diener, E., Emmons, R.A., Larsen, R.J., \& Griffin, S. (1985). The Satisfaction with Life Scale. Journal of Personality Assessment, 49, 71-75.

Ekman, P. (1994). All emotions are basic. In P. Ekman \& R.J. Davidson (Eds.), The nature of emotions. Fundamental questions. NY: Oxford University Press.

Fornell, C., \& Larcker, D.F. (1981). Evaluating structural equation models with unobserved variables and measurement error. JMR, Journal of Marketing Research, 18, 39-50.

Fredrickson, B.L. (1998). What good are positive emotions? Review of General Psychology, 2, 300-319.

Fredrickson, B.L. (2001). The role of positive emotions in positive psychology. The broadenand-build theory of positive emotions. The American Psychologist, 56, 218-226.

Fredrickson, B.L. (2005). Positive emotions. In C.R. Snyder \& S.J. Lopez (Eds.), Handbook of positive psychology (pp. 120-147). New York: Oxford University Press, Inc.

Fredrickson, B.L., \& Branigan, C.A. (2005). Positive emotions broaden the scope of attention and thought-action repertoires. Cognition and Emotion, 19, 313-332.

Fredrickson, B.L., Mancuso, R.A., Branigan, C., \& Tugade, M.M. (2000). The undoing effect of positive emotions. Motivation and Emotion, 24, 237-258. 
Gould, D., Lauer, L., Rolo, C., Jannes, C., \& Pennisi, N. (2006). Understanding the role parents play in tennis success: a national survey of junior tennis coaches. British Journal of Sports Medicine, 40, 632-636.

Gross, J.J. (1999). Emotion and emotion regulation. In L.A. Pervin \& O.P. John (Eds.), Handbook of personality (2nd ed., pp. 525-552). New York: Guilford Press.

Hanin, Y. (Ed.). (2000). Emotions in sport. Champaign, IL: Human Kinetics.

Henderick, S., \& Henderick, C. (2005). Love. In C.R. Snyder \& S.J. Lopez (Eds.), Handbook of positive psychology (pp. 472-484). NY: Oxford University Press.

Hu, L., \& Bentler, P.M. (1998). Fit indices in covariance structure modeling: Sensitivity to underparameterized model misspecifications. Psychological Methods, 4, 424-453.

$\mathrm{Hu}, \mathrm{L} .$, \& Bentler, P.M. (1999). Cutoff criteria for fit indexes in covariance structure analysis: Conventional criteria versus new alternatives. Structural Equation Modeling, 6, 1-55.

Jones, M.V., Lane, A.M., Bray, S.R., Uphill, M., \& Catlin, J. (2005). Development and validation of the Sport Emotion Questionnaire. Journal of Sport \& Exercise Psychology, 27, 407-431.

Kellmann, M., \& Kallus, K.W. (2001). Recovery-Stress Questionnaire for athletes: User manual. Champaign, IL: Human Kinetics.

Kenttä, G., \& Dieffenbach, K. (2008). Athlete perceptions of the role of recovery in elite training: Three case studies. Proceedings from The $23^{\text {th }}$ Annual Conference of the Association for the Advancement of Applied Sport Psychology. St. Louis, MO, USA: Association for the Advancement of Applied Sport Psychology.

Kenttä, G., \& Hassmén, P. (1998). Overtraining and recovery: A conceptual model. Sports Medicine (Auckland, N.Z.), 26, 1-16.

Kenttä, G., \& Hassmén, P. (2002). Underrecovery and overtraining: A conceptual model. In M. Kellmann (Ed.), Enhancing recovery: Preventing underperformance in athletes (pp. 57-77). Champaign, IL: Human Kinetics.

Kenttä, G., Hassmén, P., \& Raglin, J.S. (2006). Mood state monitoring of training and recovery in elite kayakers. European Journal of Sport Science, 4, 245-253.

Keyes, C.L.M., \& Magyar-Moe, J.L. (2002). The measurement and utility of adult subjective well-being. In S.J. Lopez \& C.R. Snyder (Eds.), Positive psychological assessment. A handbook of models and measures (pp. 411-425). Washington, DC: American Psychological Association.

Lane, A., \& Terry, P.C. (1998). Development of normative data for profile of mood states-C among adult and young athletes. Journal of Sports Sciences, 16, 94-95.

Lavallee, D., Thatcher, J., \& Jones, M.V. (Eds.). (2005). Coping and emotion in sport. Hauppauge, NY: Nova Science.

Lazarus, R.S. (2000). How emotions influence performance in competitive sport. The Sport Psychologist, 14, 229-252.

Li, F., \& Harmer, P. (1996). Confirmatory factor analysis of the Group Environmental Questionnaire with an intercollegiate sample. Journal of Sport \& Exercise Psychology, 18, 49-63.

Lucas, R.E., Diener, E., \& Larsen, R.J. (2003). Measuring positive emotions. In S.J. Lopez \& C.R. Snyder (Eds.), Positive psychological assessment. A handbook of models and measures (pp. 201-218). Washington, DC: American Psychological Association.

Main, L., \& Groove, R.J. (2009). A multi-component assessment model for monitoring training distress among athletes. European Journal of Sport Science, 4, 195-202.

Mohiyeddini, C., Pauli, R., \& Bauer, S. (2009). The role of emotion in bridging the intentionbehaviour gap: The case of sports participation. Psychology of Sport and Exercise, 10, 226-234. 
Nakamura, J., \& Csikszentmihalyi, M. (2005). The concept of flow. In C.R. Snyder \& S.J. Lopez (Eds.), Handbook of positive psychology (pp. 89-105). New York: Oxford University Press, Inc.

McNair, D., Lorr, M., \& Droppelman, L. (1992). Profile of mood states manual. San Diego: Educational and Industrial Testing Service.

Nix, G.A., Ryan, R.M., Manly, J.B., \& Deci, E.L. (1999). Revitalization through selfregulation: The effects of autonomous and controlled motivation on happiness and vitality. Journal of Experimental Social Psychology, 35, 266-284.

O'Connor, P.J. (2004). Evaluation of four highly cited energy and fatigue mood measures. Journal of Psychosomatic Research, 57, 435-441.

Raglin, J.S. (2001). Psychological factors in sport performance - The mental health model revisited. Sports Medicine (Auckland, N.Z.), 12, 875-890.

Ryan, R.M., \& Deci, E.L. (2008). From ego depletion to vitality: Theory and findings concerning the facilitation of energy available to the self. Social and Psychology Compass, 2, 702-717.

Ryan, R.M., \& Frederick, C. (1997). On energy, personality, and health: Subjective vitality as a dynamic reflection of well-being. Journal of Personality, 65, 529-565.

Soares, I., Lemos, M.S., \& Almeida, C. (2005). Attachment and motivational strategies in adolescence. Adolescence, 40, 129-154.

Sonnentag, S., \& Fritz, C. (2007). The Recovery Experience Questionnaire: Development and validation of a measure for assessing recuperation and unwinding from work. Journal of Occupational Health Psychology, 12, 204-221.

Strean, W.B., \& Strozzi-Heckler, R. (2009). (The) Body (of) knowledge: Somatic contributions to the practice of sport psychology. Journal of Applied Sport Psychology, 21, 91-98.

Tugade, M.M., \& Fredrickson, B.L. (2004). Resilient individuals use positive emotions to bounce back from negative emotional experiences. Journal of Personality and Social Psychology, 86, 320-333.

Urhausen, A., \& Kindermann, W. (2002). Diagnosis of overtraining - What tools do we have? Sports Medicine (Auckland, N.Z.), 32, 95-102. 
Appendix Instructions to EmRecQ: Below is a list of words that describe a range of feelings that any person can experience. Please read each one and carefully indicate on the scale for each item how you feel right now. There are no right or wrong answers.

\begin{tabular}{lccccc}
\hline & $\begin{array}{c}\text { Not at } \\
\text { all }\end{array}$ & & & & Extremely \\
\hline 1. På gott humör (In high spirits) & 1 & 2 & 3 & 4 & 5 \\
2. Trygg (Secure) & 1 & 2 & 3 & 4 & 5 \\
3. I balans (Well balanced) & 1 & 2 & 3 & 4 & 5 \\
4. Uppskattad (Appreciated) & 1 & 2 & 3 & 4 & 5 \\
5. Energisk (Energetic/vigorous) & 1 & 2 & 3 & 4 & 5 \\
6. Lycklig (Happy) & 1 & 2 & 3 & 4 & 5 \\
7. Har kontroll (In control) & 1 & 2 & 3 & 4 & 5 \\
8. Inre lugn (Inner calm) & 1 & 2 & 3 & 4 & 5 \\
9. Omtyckt (Popular/well liked) & 1 & 2 & 3 & 4 & 5 \\
10. Pigg (Alert) & 1 & 2 & 3 & 4 & 5 \\
11. Glad (Joyful) & 1 & 2 & 3 & 4 & 5 \\
12. Stabil (Stable) & 1 & 2 & 3 & 4 & 5 \\
13. Harmonisk (At peace) & 1 & 2 & 3 & 4 & 5 \\
14. Tillhörighet (Belonging) & 1 & 2 & 3 & 4 & 5 \\
15. Utvilad (Well-rested) & 1 & 2 & 3 & 4 & 5 \\
16. Munter (Cheerful) & 1 & 2 & 3 & 4 & 5 \\
17. Säker (Safe/certain) & 1 & 2 & 3 & 4 & 5 \\
18. Tillfreds (Well-satisfied) & 1 & 2 & 3 & 4 & 5 \\
19. Närhet med andra (Close to others) & 1 & 2 & 3 & 4 & 5 \\
20. Uppladdad (Fired up) & 1 & 2 & 3 & 4 & 5 \\
21. Behagligt nöjd (Pleased) & 1 & 2 & 3 & 4 & 5 \\
22. Stark (Strong) & 1 & 2 & 3 & 4 & 5 \\
\hline & & & & & \\
\hline & 1 & 2 & & 4 \\
\hline
\end{tabular}

